\title{
Coagulação intravascular disseminada e COVID-19: mecanismos fisiopatológicos
}

\author{
Disseminated intravascular coagulation and COVID-19: pathophysiological mechanisms
}

Marco Orsini ${ }^{\dagger *}, \neq^{*}$, Jacqueline Stephanie Fernandes do Nascimento ${ }^{\ddagger}$, Nicolle dos Santos Moraes Nunes ${ }^{\ddagger}$, Janie Kelly Fernandes do Nascimento ${ }^{\ddagger}$, Marco Azizi ${ }^{\ddagger}$, Carlos Eduardo Cardoso ${ }^{\dagger}$

Como citar esse artigo. Orsini,

M.; Do Nascimento, J.S.F.; Nunes, N.S.M.; Do Nascimento, J.K.F.; Azizi, M.; Cardoso, C.E. Coagulação intravascular disseminada e COVID-19: mecanismos fisiopatológicos. Revista de Saúde. 2020 Jan./Jun.; 11 (1): 87-90.

\section{Resumo}

A coagulação intravascular disseminada (CIVD) é, atualmente, definida como uma síndrome adquirida, caracterizada pela ativação difusa da coagulação intravascular, levando à formação e deposição de fibrina na microvasculatura. Há relatos de coagulopatia relacionada à CCOVID-19, doença causada pelo novo coronavírus SARS-CoV-2. Foi realizada a atualização da literatura acerca da associação entre COVID-19 e a presença de CIVD nas bases dados LIlacs, Bireme, Pubmed (inglês e português) no presente ano. A Coagulação Intravascular disseminada está frequentemente associada à doenças infecciosas que levam à quadros de septicemia. A literatura vigente descreve que a forma grave da COVID-19 é frequentemente complicada com coagulopatia. A terapia anticoagulante tem sido bastante implementada em pacientes com apresentação grave de COVID-19 que atendem aos critérios de coagulopatia induzida pela sepse e/ou dímero-D em níveis aumentados em concomitância a demais biomarcadores que indiquem gravidade, sem contraindicações à anticoagulação. Até o presente momento não existem recomendações precisas em relação ao manejo dos pacientes com coagulopatia por COVID-19. Além disso, são escassos os dados na literatura que associam o uso de anticoagulantes ou antiplaquetários à ocorrência ou gravidade da COVID-19.

Palavras-chave: COVID-19; Coagulopatia; Fisiopatologia; Coagulação Intravascular disseminada.

\begin{abstract}
Disseminated intravascular coagulation (DIC) is currently defined as an acquired syndrome, characterized by the diffuse activation of intravascular coagulation, leading to the formation and deposition of fibrin in the microvasculature. There are reports of coagulopathy related to COVID-19, the disease caused by the new coronavirus SARS-CoV-2. The literature update about the association among COVID-19 and the presence of DIC in the LIlacs, Bireme, Pubmed (English and Portuguese) databases this year. Disseminated intravascular coagulation is often associated with infectious diseases that lead to septicemia. Current literature describes that the severe form of COVID-19 is often complicated by coagulopathy. Anticoagulant therapy has been widely implemented in patients with severe presentation of COVID-19 that meet the criteria for coagulopathy induced by sepsis and / or D-dimer at increased levels simultaneously with other biomarkers that indicate severity, without contraindications to anticoagulation. To date, there are no precise recommendations regarding the management of patients with COVID-19 coagulopathy. In addition, there is little data in the literature associating the use of anticoagulants or antiplatelet agents with the occurrence or severity of COVID-19.
\end{abstract}

Keywords: COVID-19; Coagulopathy; Pathophysiology; Disseminated intravascular coagulation.

\section{Introdução}

A COVID-19 no momento presente acomete mais de um milhão de pessoas, e o número de casos notificados aumenta paulatinamente em vários países. Devido à rápida disseminação e dificuldade no controle da patologia foi declarada situação de pandemia pela Organização Mundial da Saúde (OMS) ${ }^{1,2}$. Os quadros clínicos apresentados pelos pacientes infectados pelo
SARS-CoV-2 variam desde indivíduos assintomáticos até quadros graves que culminam em óbito.

A coagulação intravascular disseminada (CIVD) é, atualmente, definida como uma síndrome adquirida, caracterizada pela ativação difusa da coagulação intravascular, levando à formação e deposição de fibrina na microvasculatura ${ }^{3}$. Tal condição é associada à diversas entidades clínicas, sobretudo infecções que levam à septicemia. ${ }^{4}$ Há relatos de coagulopatia 
relacionada à COVID-19, doença pelo novo coronavírus SARS-CoV-2.

Segundo pesquisadores e especialistas, fundamentados em experiência clínica e investigação de alguns poucos estudos descritivos, é importante ressaltar o papel da condição de hipercoagulabilidade na fisiopatologia da COVID-19, tendo em vista que a elevação do dímero-D, um produto da degradação de fibrina, ocorre progressivamente com o agravamento da infecção. Quando em níveis altos, o dímero-D tem sido frequentemente relacionado a um aumento na taxa de mortalidade 5 .

Devido ao curto período de tempo que a COVID19 levou para gerar a maior crise de saúde do século, pouco ainda se sabe sobre ela. No entanto, a falta ou demora de assistência médica pode resultar em altas taxas de óbito. Sendo assim, é fundamental que se conheça os mecanismos fisiopatológicos que levam à complicações do quadro. O objetivo do presente estudo é atualizar conhecimentos relacionados à fisiopatologia da CIVD em pacientes infectados por SARS-CoV-2 com base numa breve revisão de literatura.

\section{Materiais e Métodos}

A proposta do presente artigo é, com base na literatura vigente, produzir e atualizar reflexões acerca do atual contexto de pandemia. Para tal, fora realizada uma busca nas principais bases de dados: LILACS, BIREME, PuBMED nos idiomas português e inglês, de artigos publicados no ano vigente. A escolha ocorreu de forma aleatória, obviamente, seguindo uma linha de raciocínio dos autores envolvidos. As palavraschave pesquisadas foram: COVID-19; Coagulopatia; Fisiopatologia; Coagulação Intravascular disseminada.

\section{Resultados}

Dos 68 estudos encontrados mediante o cruzamento dos descritores acima, 16 foram escolhidos pelos autores de forma aleatória, apenas seguindo uma linha de raciocínio lógico. Desses, nenhum artigo fora excluído remanescendo 16 artigos que compuseram a amostra final.

\section{Discussão}

A Coagulação Intravascular disseminada está frequentemente associada à doenças infecciosas que levam à quadros de septicemia. A literatura vigente descreve que a forma grave da COVID-19 é frequentemente complicada com coagulopatia. Dentro desse contexto, a CIVD é relatada em grande parte dos óbitos ${ }^{6,7}$.
Em torno do $7^{\circ}$ ao $12^{\circ}$ dia dos sintomas transcorrem as complicações ${ }^{8,9}$. A manifestação grave da infecção é marcada por uma resposta inflamatória imunológica acentuada, caracterizada pela presença de monócitos, linfócitos, neutrófilos e macrófagos ${ }^{10}$. Após procedimentos de autópsia pouco invasivos foram evidenciados dano alveolar difuso, infiltrado inflamatório intersticial mononuclear com trombose em microcirculação e formação de membrana hialina ${ }^{11}$. Esses pacientes apresentaram altos índices de citocinas pró-inflamatórias no sistema circulatório, como interferon- $g$, interleucinas 1 e 6 e fator de necrose tumoral - TNF, formando o conceito de tempestade de citocinas ${ }^{1}$.

Injúrias fora do âmbito do aparelho respiratório, bem como trombose podem acontecer sem que haja assiduidade local confirmada do vírus, admitindo que a infecção pelo SARS-CoV-2 compreenda uma severa resposta inflamatória, com estado de hipercoagulabilidade, isquemia e exacerbado por hipoxemia ${ }^{11,9,12}$

Pacientes que apresentem infecção e consequentemente desenvolvem resposta inflamatória sistêmica podem evoluir com lesão endotelial e consecutivo aumento na geração de trombina e diminuição da fibrinólise endógena ${ }^{13,14}$. Pode-se assim denominar o estado pró-trombótico de Coagulopatia Induzida pela Sepse (SIC) que antecede a CIVD ${ }^{15,16}$. A fisiologia que rege a SIC finaliza em uma condição próhemostática. Tem-se estudado que os elementos chaves que interferem no distúrbio de coagulação no decorrer da sepse são as citocinas inflamatórias.

Indivíduos que são acometidos por infecções virais estão sujeitos a evoluir para sepse associada à disfunção orgânica. A sepse está bem especificada como um dos fatores causais mais comum da CIVD, que se desenvolve com a liberação de citocinas pró inflamatórias, como fator de Von Willebrand e expressão do fator tecidual, que ativam monócitos e células endoteliais. A trombina circulante, não impedida pela via fisiológica anticoagulante, pode ativar as plaquetas e incitar a fibrinólise ${ }^{17,18}$.

O distúrbio ocorrido nas células endoteliais provocado pela infecção sucede em uma intensa geração de trombina e na redução da fibrinólise, apontando para um estado hipercoagulável em pacientes diagnosticados com infecção ${ }^{13,14}$ como na COVID-19. Ademais, o estado de hipóxia gerado na forma grave da patologia pode estimular a trombose, pelo aumento da viscosidade sanguínea e por uma via de sinalização dependente do fator de transcrição provocado por hipóxia ${ }^{6}$.

Estudos mostram relação cruzada entre inflamação e coagulação, sendo a inflamação responsável por provocar a ativação da coagulação. A coagulação por sua vez intensifica a atividade inflamatória ${ }^{19,20}$. A coagulação no período da sepse sofre distúrbios; e as plaquetas 
possuem responsabilidade central por esse evento, sendo ativadas por mediadores pró-inflamatórios, como o fator ativador de plaquetas, além da trombina gerada. Fora do estado patológico, há um controle da ativação da coagulação por três vias anticoagulantes: sistema ativado da proteína $\mathrm{C}$, inibidor da via do fator tecidual e o sistema antitrombina. No entanto, essas vias fisiológicas sofrem alterações na sepse. Perante esse distúrbio do sistema de coagulação, há uma minimização do sistema de coagulação. ${ }^{21,22}$

Dentro desse contexto, a literatura vigente associa o estado de hipercoagulabilidade à apresentação grave da COVID-19, em que SIC e/ou CIVD se demonstraram presentes em grande parte dos $\operatorname{casos}^{23}$. A terapia anticoagulante tem sido bastante implementada em pacientes com apresentação grave de COVID-19 que atendem aos critérios de SIC e/ou dímero-D em níveis aumentados concomitantemente a demais biomarcadores que indiquem gravidade, sem contraindicações à anticoagulação. Sendo assim, ela pode ser enquadrada como alternativa terapêutica na opinião de especialistas.

O provável benefício de se minimizar o estado de hipercoagulabilidade deve ser equilibrado com o risco de sangramento. É necessário também destacar que, ao abordar anticoagulação, a heparina de baixo peso molecular (HBPM) é o fármaco de escolha para pacientes estabilizados e com depuração de creatinina normal. Ainda assim, não existem resultados suficientes para definir critérios importantes à preparação do plano terapêutico. Dessa forma, estudos prospectivos que abordem os principais critérios indicadores de terapia anticoagulante em pacientes graves com COVID-19 tornam-se fundamentais para apoiar sua aplicabilidade clínica. ${ }^{1}$

\section{Conclusão}

A fisiopatologia da COVID-19 abrange a intensa ativação do processo inflamatório e incitamento do sistema trombótico, ocasionando assim a complicação clínica de Coagulação Intravascular Disseminada, que está relacionada com a maioria dos casos de óbitos. Atualmente, especialistas sugerem a terapia anticoagulante para pacientes críticos, que possuam critérios para SIC ou elevação de dímero-D. Mais estudos são necessários, afim de se corroborar o papel da anticoagulação na prevenção de complicações da COVID-19.

\section{Referências}

1. Nascimento JHP, Gomes BFO, Júnior PRC, Petriz JL, Rizk SI, Costa IBSS, et al. COVID-19 e Estado de Hipercoagulabilidade: Uma Nova Perspectiva Terapêutica. Sociedade Brasileira de Cardiologia. 2020. DOI: https://doi. org/10.36660/abc.20200308
2. Organização Mundial da Saúde. Relatórios de situação da doença coronavírus 2019 (COVID-2019). Disponível em: <https:/www.who.int/ emergencies/diseases/novel-coronavirus-2019/> acesso em: 29/04/2020.

3. Levi M, Ten cate H. Disseminated intravascular coagulation. N Engl J Med. 199, 341, 586-592.

4. Pintão MC, Franco R. Coagulação intravascular disseminada. Universidade de São Paulo. 2001; 34: 282-91. DOI: https://doi.org/10.11606/issn.21767262.v34i3/4p282-291

5. Chen N, Zhou M, Dong X, Qu J, Gong F, Han Y, et al. Epidemiological and clinical characteristics of 99 cases of 2019 novel coronavirus pneumonia in Wuhan, China: a descriptive study. Lancet. 2020; 395; 10223; 507. DOI: https://doi.org/10.1016/S0140-6736(20)30211-7

6. Tang N, Bai H, Chen X, Gong J, Li D, Sun Z. Anticoagulant treatment is associated with decreased mortality in severe coronavírusdesease 2019 patients with coagulopathy. J ThrombHaemost. 2020; 18; 1094-1099. https:// doi.org/10.1111/jth.14817

7. Singer M, Deutschman CS.; SEYMOUR, C.W.; et al. The third international consensus definitions for sepsis and septic shock (Sepsis-3). JAMA. 2016;315(8):801-810.

8. Huang C, Wang Y, Li X, Ren L, Zhao J, Hu Y, et al. Clinical features of patients infected with 2019 novel coronavirus in Wuhan, China. Lancet. 2020; 395 (10223); 497-506. https://doi.org/10.1016/ S0140-6736(20)30183-5

9. Wang D, Hu B, Hu C, Zhu F, Liu X, Zhang J, et al. Clinical Characteristics of 138 Hospitalized Patients With 2019 Novel Coronavirus-Infected Pneumonia in Wuhan, China. JAMA. 2020; 323 (11); 1061-1069. doi:10.1001/jama.2020.1585

10. Chen G, Wu D, Guo W, Li X, Sun Y, Li J, et al. J Clinical and immunologic features in severe and moderate Coronavirus Disease 2019. J Clin Invest. 2020. https://doi.org/10.1172/JCI137244.

11. Zhang T, Sun LX, Feng RE. Comparison of clinical and pathological features between severe acute respiratory syndrome and coronavirus disease 2019. ZhonghuaJie He He Hu Xi Za Zhi. 2020; 43 (0). DOI: 10.3760/ cma.j.cn112147-20200311-00312.

12. Luo W, Yu H, Gou J, Li X, Sun Y, Li J, et al. Clinical Pathology of Critical Patient with Novel Coronavirus Pneumonia (COVID-19). Preprints. 2020, 2020020407

13. Levi M, Van der poll T. Coagulation and sepsis. Thromb Res. 2017; 149; $38-44$

14. Schmitt FCF, Manolov V, Morgenstern J, Fleming T, Heitmeier S, Ubile $\mathrm{F}$, et al. Acute fibrinolysis shutdown occurs early in septic shock and is associated with increased morbidity and mortality: results of an observational pilot study. Ann Intensive Care. 2019; 9(1); 19. DOI: 10.1186/s13613-0190499-6

15. Iba T, Levy JH, Warkentin TE, Tachill J, Van der poll T. Diagnosis and management of sepsis-induced coagulopathy and disseminated intravascular coagulation. J ThrombHaemost. 2019; 17 (11); 1989-94. doi: 10.1111/ jth. 14578

16. Iba T, Nisio MD, Levy JH, Kitamura N, Thachil J. New criteria for sepsis-induced coagulopathy (SIC) following the revised sepsis definition: a retrospective analysis of a nationwide survey. BMJ Open. 2017; 7(9); e017046. http://dx.doi.org/10.1136/bmjopen-2017-017046

17. Kitchens CS. Thrombocytopenia and thrombosis in disseminated intravascular coagulation (DIC). Educ Program Am Soc Hematol. 2009; 2009(1); 240- 246. https://doi.org/10.1182/asheducation-2009.1.240

18. Tang N, Li D, Wang X, Sun Z. Abnormal coagulation parameters are associated with poor prognosis in patients with novel coronavirus pneumonia. J ThrombHaemost. 2020; 18: 844-847. https://doi.org/10.1111/jth14768

19. Levi AM, Van der poll HR, Buller. The bidirectional relationship between coagulation and inflammation, Circulation. 2004; 109(22); 2698-704.

20. Zimmerman BGA, Mcintyre TM, Prescott SM, Stafforini S. The plateletactivating factor signaling system and its regulators in syndromes of inflammation and thrombosis. Crit Care Med. 2002; 30; S294-S301. DOI: 10.1097/00003246-200205001-00020 
21. Shebuski CRJ, Kilgore KS. Role of inflammatory mediators in thrombogenesis. J Pharmacol Exp Ther. 2002; 300(3); 729-35. DOI: 10.1124/ jpet.300.3.729

22. Levi DM, Van der poll T. Coagulation in patients with severe sepsis. Semin. Thromb. Hemost. 2015; 41(1); 9-15.

23. Zhang Y, Cao W, Xiao M, Li YJ, Yang Y, Zhao J, et al. Clinical and coagulation characteristics of 7 patients with critical COVID-2019 pneumonia and acro-ischemia. ZhonghuaXue Ye Xue Za Zhi. 2020; 28; 41(0); E006 